\title{
Mesoporous hybrid materials by simultaneous pseudomorphic transformation and functionalization of silica microspheres
}

Michael J. Reber and Dominik Brühwiler*

Institute of Chemistry and Biological Chemistry

Zürich University of Applied Sciences

CH-8820 Wädenswil, Switzerland

* Corresponding author: dominik.bruehwiler@zhaw.ch

This is the peer reviewed version of the following article: Mesoporous Hybrid Materials by Simultaneous Pseudomorphic Transformation and Functionalization of Silica Microspheres, M. J. Reber, D. Brühwiler, Particle \& Particle Systems Characterization 32 (2015) 243, which has been published in final form at http://dx.doi.org/10.1002/ppsc.201400150. This article may be used for non-commercial purposes in accordance with Wiley Terms and Conditions for Self-Archiving. 


\begin{abstract}
Non-agglomerated amino-functionalized mesoporous silica microspheres are synthesized by a one-pot synthesis from a parent silica material. Narrow pore size distributions in the range from 3 to $5 \mathrm{~nm}$ are obtained with alkyltrimethylammonium structure-directing agents. By following the pseudomorphic transformation pathway, the particle size distribution and spherical morphology of the parent silica are retained during the synthesis. The products contain accessible and uniformly distributed amino groups. The average pore size and the ratio of small uniform mesopores $(<5 \mathrm{~nm})$ to larger mesopores and macropores can be controlled by choosing the appropriate structure-directing agent and by adjusting the concentration of the amino-functionalized alkoxysilane precursor, leading to a variety of mesomacroporous hybrid materials.
\end{abstract}




\section{Introduction}

The functionalization of mesoporous silica particles with organic moieties has received increased attention in a variety of research areas. Functional groups on the pore surface can regulate the release of molecular cargo in drug delivery applications, ${ }^{[1,2]}$ catalyze reactions, ${ }^{[3]}$ or specifically adsorb target species. ${ }^{[4]}$ Moieties on the external particle surface can be employed to adjust properties such as dispersibility, ${ }^{[5]}$ cellular interactions, ${ }^{[6,7]}$ or biodistribution. ${ }^{[8,9]}$ Functionalization of mesoporous silica is typically achieved by postsynthetic treatment (grafting) or by direct synthesis (co-condensation). ${ }^{[10]}$ Postsynthetic modification introduces the functional groups after the formation of the mesoporous silica. The distribution of the functional groups on the surface can be controlled to a certain extent, but achieving a uniform distribution over the entire pore surface tends to be difficult, as grafting occurs preferentially on the most accessible sites, i.e. on the external particle surface and on the pore surface close to the pore entrances. ${ }^{[1,12]}$ Particularly in the case of microparticles and pore radii in the size range of the molecule to be grafted, the accessibility of the pores becomes an issue, causing non-uniform distributions of surface-grafted functional moieties. ${ }^{[13]}$ Co-condensation circumvents this problem by introducing the organic functionalities during the formation of the mesoporous silica framework. However, application of the co-condensation pathway requires sufficient stability of the organic moiety under the often strongly alkaline or acidic hydrothermal synthesis conditions of the mesoporous silica. Furthermore, high functionalization degrees result in decreasing mesoscopic order (broad pore size distributions) with an additional effect on particle size and shape. ${ }^{[14-16]}$

A distinct pore diameter, a defined particle size and shape, as well as a controllable degree of functionalization are crucial parameters regarding the application of mesoporous silica in catalysis, chromatography, or drug delivery. The development of simple and robust one-pot methods that allow for a simultaneous adjustment of these parameters is challenging. An attractive method to prepare mesoporous silica with a desired particle size and shape is based on pseudomorphic transformation. ${ }^{[17-20]}$ 
This concept uses pre-shaped silica particles which are gradually dissolved and simultaneously reprecipitated in the presence of a structure-directing agent (SDA), thereby preserving their initial size and shape. Each particle is thought of acting as a microreactor in which silica is dissolved and rearranged by the interaction with the SDA. The adjustment of the pore size of mesoporous silica typically involves the use of different SDAs, also in combination with swelling agents. ${ }^{[21]}$ Pore size tuning by the addition of swelling agents has been successfully applied to the pseudomorphic transformation pathway. ${ }^{[22]}$

The simultaneous control of particle shape and functionalization degree in one-pot reactions is problematic. In conventional co-condensation, the amount of organic precursor, often an organotrialkoxysilane, can have a substantial influence on the final shape and size of the functionalized mesoporous silica particles. ${ }^{[14-16]}$ We have therefore developed a synthesis method that combines the concepts of pseudomorphic transformation and co-condensation, allowing the preparation of nonagglomerated functionalized MCM-41 type mesoporous silica microspheres.

\section{Results and Discussion}

\subsection{General Concept}

The general concept of the simultaneous pseudomorphic transformation and functionalization as schematically shown in Figure 1 includes the control of the pore size, while retaining the particle morphology and establishing organic functionalities on the mesopore surface. We have investigated the control of the pore size by means of different alkyltrimethylammonium SDAs and further developed this principle by adding 3-aminopropyltriethoxysilane (APTES) during the transformation, which ultimately allows the simultaneous incorporation of functional groups into the mesoporous silica framework. 


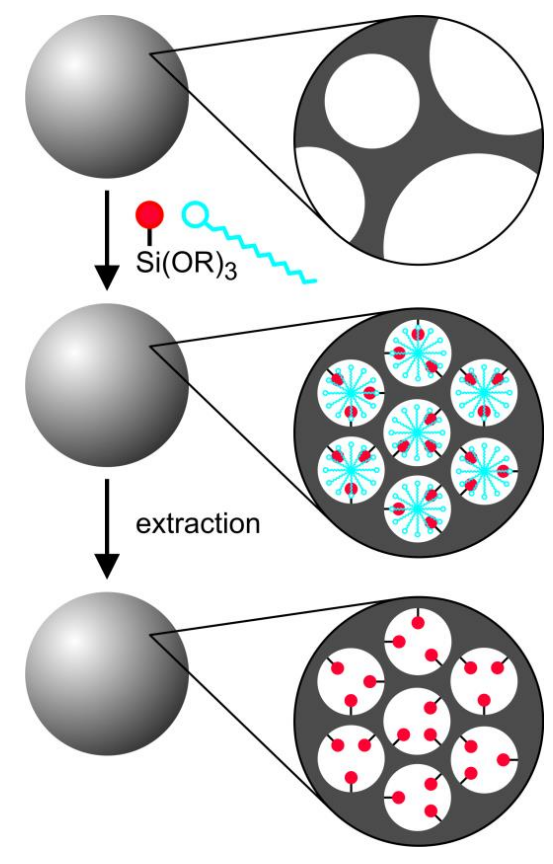

Figure 1. General concept of pseudomorphic transformation and simultaneous functionalization of porous silica microspheres. Hydrothermal treatment of an alkaline aqueous solution containing a structure-directing agent (SDA, blue), an organotrialkoxysilane bearing a functional group (red), and a parent silica material yields a functionalized mesoporous material with a narrow pore size distribution and an unaltered particle morphology.

The purely siliceous products are designated as Sq (extracted) and Sqc (calcined), where $q$ indicates the alkyl chain length of the SDA used in the pseudomorphic transformation (e.g. $q=16$ for hexadecyltrimethylammonium bromide, CTAB). Sample names of the amino-functionalized materials additionally include information about the amount of APTES added to the reaction mixture and are therefore designated as $\mathrm{S} q \mathrm{~A}(r)$, where $r$ specifies the amount of APTES in units of mmol per gram of silica.

Pseudomorphic transformation of Kromasil silica with CTAB converts the broad pore size distribution (PSD) of the parent material into a narrow PSD centered at $4.15 \mathrm{~nm}(\mathrm{FWHM}=0.55 \mathrm{~nm})$ with only small contributions from parental pores (Figure 2). In the following, the small mesopores with pore sizes in the range between 2 and $5 \mathrm{~nm}$ will be referred to as primary mesopores. Comparison of the primary 
mesopore volume $\left(V_{p}\right)$ to the total pore volume $\left(V_{\text {tot }}\right)$ indicates a primary mesopore volume fraction of $84 \%$ (Table 1, sample S16). The transformation is accompanied by an increase of the BET surface area $\left(S_{B E T}\right)$ from an initial value of $93 \mathrm{~m}^{2} \mathrm{~g}^{-1}$ to $771 \mathrm{~m}^{2} \mathrm{~g}^{-1}$. The total pore volume remains roughly the same during the transformation. When the SDA is removed by calcination instead of extraction, contraction of the mesoporous silica framework leads to slightly decreased values for the pore diameter, BET surface area, and pore volume (Table 1, sample S16c).

We have recently reported that the external particle surface of MCM-41 and SBA-15 type mesoporous silica can be selectively functionalized by using 3-aminopropyltris(methoxyethoxyethoxy)silane (APTMEES). ${ }^{[11]}$ Subsequent coupling of a fluorescent label such as fluorescein 5 -isothiocyanate (FITC) to the grafted amino groups and imaging by confocal laser scanning microscopy (CLSM) revealed the exclusive presence of the labels on the external particle surface with the pore surface remaining fully accessible. Applying this method to Kromasil silica does not result in a selective functionalization of the external surface (Figure 2, CLSM image A), because the APTMEES molecules are able to access the entire surface via the macropores. The method is, however, successful after pseudomorphic transformation due to the increased and less accessible surface area obtained through the formation of the primary mesopores. The external surface functionalization with APTMEES can be either directly performed on the calcined pseudomorphically transformed materials (e.g. S16c, Figure 2, CLSM image B) or on the assynthesized materials (e.g. S16 before SDA extraction, Figure 2, CLSM image C). Both methods yield an excellent selectivity for the external surface, thereby opening possibilities to adjust the interaction of the particles with their environment, including strategies for optimizing the dispersibility in various solvents. 

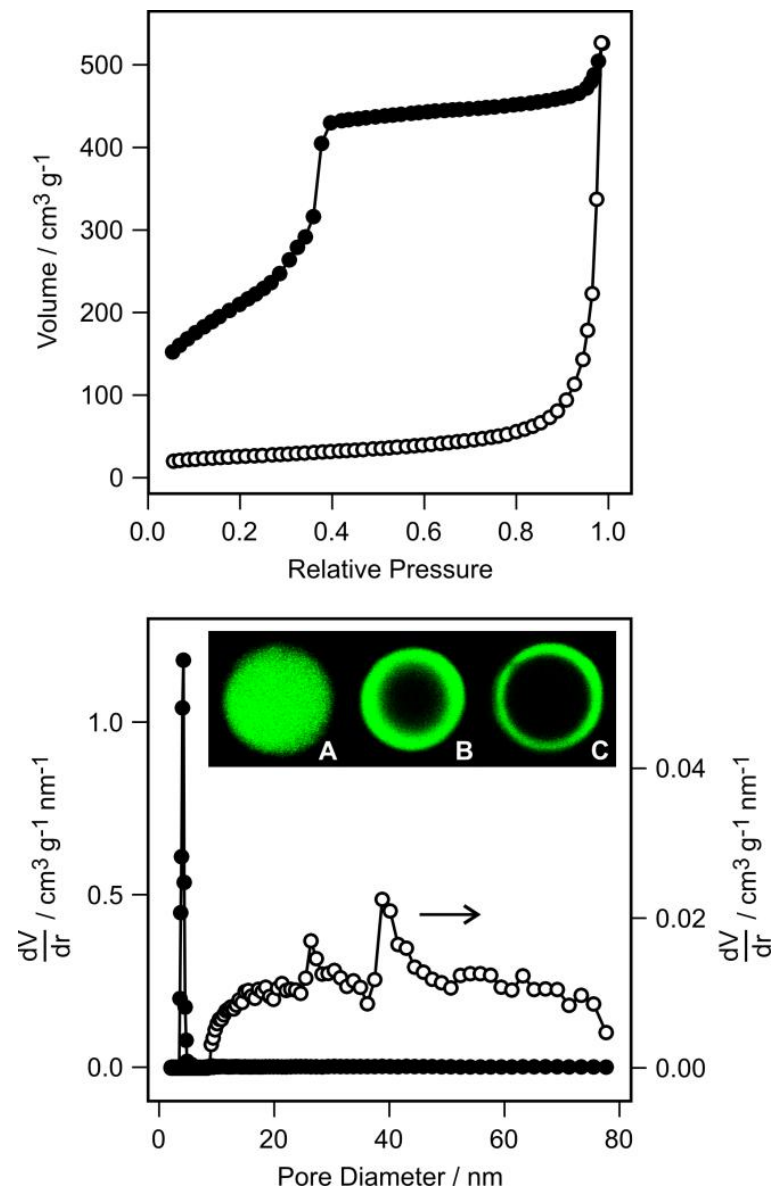

Figure 2. Nitrogen adsorption isotherms (top) and PSDs (bottom) of Kromasil silica (empty circles) and the pseudomorphically transformed product S16 (filled circles). The inset shows representative CLSM images of APTMEES/FITC-functionalized Kromasil silica (A), S16c (B), and S16 (C). Optical slices through the center of the microparticles were selected. 
Table 1. Properties of the purely siliceous materials.

\begin{tabular}{|c|c|c|c|c|c|c|}
\hline Sample & $\begin{array}{l}\mathrm{d}_{\mathrm{DFT}}{ }^{\mathrm{a})} \\
{[\mathrm{nm}]}\end{array}$ & $\begin{array}{c}\text { FWHM }^{\text {b) }} \\
{[\mathrm{nm}]}\end{array}$ & $\begin{array}{c}\mathrm{S}_{\mathrm{BET}} \\
{\left[\mathrm{m}^{2} \mathrm{~g}^{-1}\right]}\end{array}$ & $\begin{array}{c}\mathrm{V}_{\mathrm{p}} \\
{\left[\mathrm{cm}^{3} \mathrm{~g}^{-1}\right]}\end{array}$ & $\begin{array}{c}V_{\text {tot }} \\
{\left[\mathrm{cm}^{3} \mathrm{~g}^{-1}\right]}\end{array}$ & $\mathrm{V}_{\mathrm{p}} / \mathrm{V}_{\text {tot }}$ \\
\hline Kromasil & - & - & 93 & 0.00 & 0.78 & 0.00 \\
\hline S10 & 3.03 & 0.35 & $-{ }^{c)}$ & 0.53 & 0.65 & 0.82 \\
\hline S10c & 2.66 & 0.43 & $-{ }^{c)}$ & 0.36 & 0.57 & 0.63 \\
\hline S12 & 3.44 & 0.42 & 819 & 0.53 & 0.76 & 0.70 \\
\hline S12c & 3.12 & 0.43 & 738 & 0.43 & 0.62 & 0.69 \\
\hline S14 & 3.96 & 0.43 & 755 & 0.57 & 0.77 & 0.74 \\
\hline S14c & 3.47 & 0.53 & 704 & 0.44 & 0.69 & 0.64 \\
\hline S16 & 4.15 & 0.55 & 771 & 0.65 & 0.77 & 0.84 \\
\hline S16c & 3.56 & 0.62 & 712 & 0.45 & 0.61 & 0.73 \\
\hline
\end{tabular}

\footnotetext{
${ }^{a)}$ Average pore diameter of the primary mesopores determined by the NLDFT method; ${ }^{\text {b) }}$ Full width at half maximum of the primary mesopore size distribution; ${ }^{\mathrm{C})}$ Capillary condensation in the BET region.
}

Particle agglomeration and broad particle size distributions are often observed in the classical synthesis of MCM-41 type materials, proving disadvantageous in applications such as chromatography, where homogeneous batches of spherical microparticles are desired. ${ }^{[23-25]}$ Pseudomorphic transformation circumvents this problem by starting with a non-agglomerated silica sample. After the transformation, the particle size distribution of the parent Kromasil silica was largely retained, and no additional particle agglomeration was observed (Figure 3). However, the products contained minor amounts of submicrometer particles with irregular morphology, indicating that nucleation of silicate species outside of the microspheres might have occurred to some degree. Additionally, a small fraction of hemispherical particles was found in the Scanning Electron Microscopy (SEM) images of the products. These fragments are most likely the result of a fast transformation leading to particle explosion. ${ }^{[22]}$ 

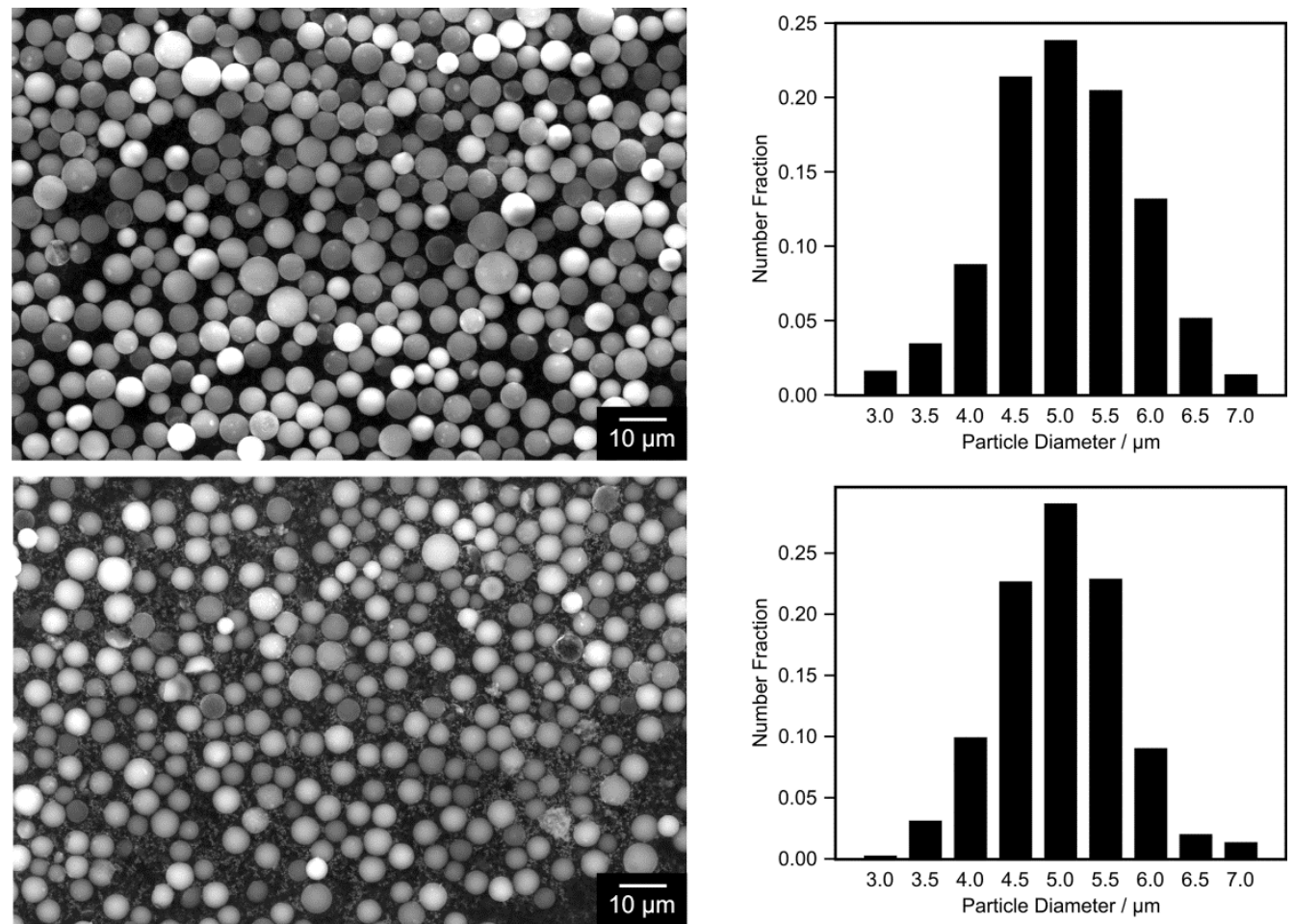

Figure 3. SEM images and particle size distributions of Kromasil silica (top) and S16 (bottom).

The pseudomorphic transformation relies on the dissolution and reprecipitation of silicate species in the porous material. To avoid the formation of mesoporous silica in the surrounding solution, the process of reprecipitation needs to occur at a rate that is equal to or faster than the rate of silica dissolution. Previous studies indicate that the final product only contains a fraction of the initial silica matter content (70\%), with the remaining fraction being dissolved during the process. ${ }^{[18]}$ The question remains to what extent an interchange of silicate species between the particles occurs during the pseudomorphic transformation. An experiment with a mixture of differently labeled starting materials (green and red labeled Kromasil silica) reveals a significant degree of interchange (Figure 4). Interestingly, this interchange already sets in at an early stage of the synthesis, i.e. before hydrothermal treatment. Removal of the surface-bound fluorescent labels by hydrolysis of the anchoring siloxane bonds and redistribution occurs before the formation of the primary mesopores by pseudomorphic transformation. 
This efficient interchange of silicate species is beneficial for the simultaneous introduction of functionalized alkoxysilanes such as APTES, as it promotes the uniform distribution of the functional groups. The pseudomorphic transformation of the mixture of red and green labeled Kromasil silica indeed yields a mesoporous product containing indistinguishable double labeled yellow particles.

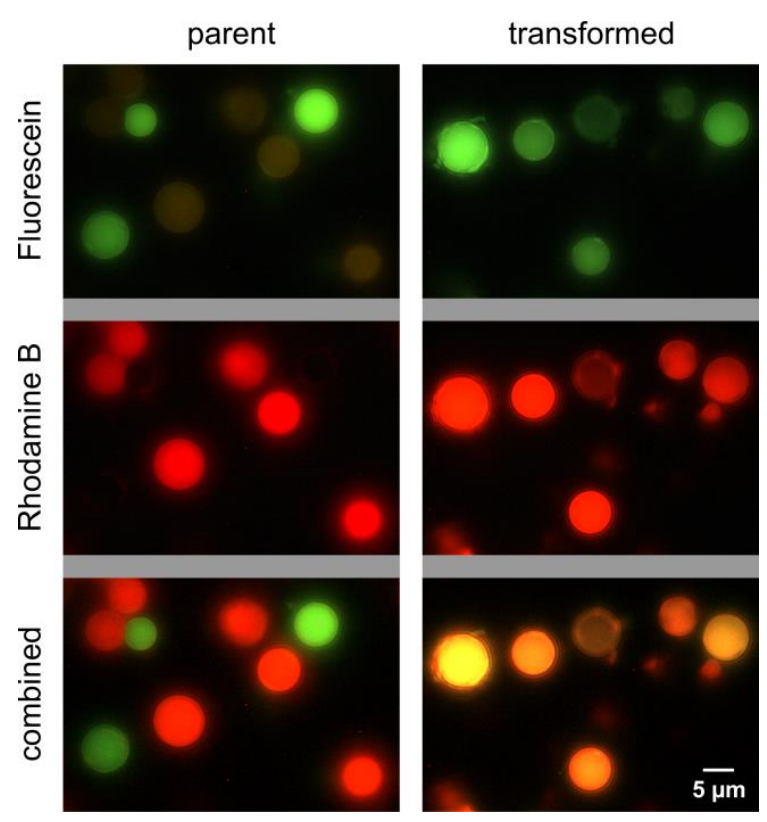

Figure 4. Fluorescence microscopy images of a mixture of red (Rhodamine B) and green (Fluorescein) labeled Kromasil particles (left column) and of the same sample after pseudomorphic transformation (right column).

\subsection{Pore Size Control}

The most obvious way of tuning the pore size of MCM-41 type materials is by changing the alkyl chain length of the alkyltrimethylammonium bromide SDAs. Reducing the alkyl chain by a $\left(\mathrm{CH}_{2}\right)_{2}$ unit decreases the pore size by roughly $0.3 \mathrm{~nm}$. This principle has been successfully applied to the conventional MCM41 synthesis pathway, using tetraethyl orthosilicate, sodium silicate, or silica gel as a silica source. ${ }^{\text {[26-31] }}$ As shown in Figure 5, adaptation to the pseudomorphic transformation of Kromasil silica yields narrow 
pore size distributions when using $\mathrm{C}_{10}, \mathrm{C}_{12}, \mathrm{C}_{14}$, and $\mathrm{C}_{16}$ alkyl chains in alkyltrimethylammonium SDAs. Type IV nitrogen sorption isotherms, typical for ordered mesoporous materials, were observed in all cases. The mode of SDA removal (calcination or extraction) has an influence on the properties of the products. Calcination results in a contraction of the silica framework through further condensation. This step is typically accompanied by a slight broadening of the PSD. Extraction led to exceptionally narrow PSDs. Pore sizes, BET surface areas, and pore volumes were slightly larger compared to the respective products obtained after calcination (Table 1). This is in agreement with earlier observations on conventionally synthesized MCM-41. ${ }^{[32]}$ As we attempt to synthesize organo-functionalized mesoporous silica, extraction is the method of choice for the removal of the SDA.

From the nitrogen sorption analysis data compiled in Table 1, the following general trends can be identified: (i) The total pore volume is largely retained after pseudomorphic transformation and extraction of the SDA, whereas the BET surface area is increased by a factor of roughly 8. (ii) Compared to SDA removal by extraction, calcination broadens the PSD and shifts it to smaller pore sizes. Furthermore the BET surface area and the pore volume are reduced by calcination. This is indicative of a contraction of the silica framework. (iii) The percentage of primary mesopore volume formed during the pseudomorphic transformation is above $70 \%$ for the extracted samples and slightly lower for the calcined samples. 


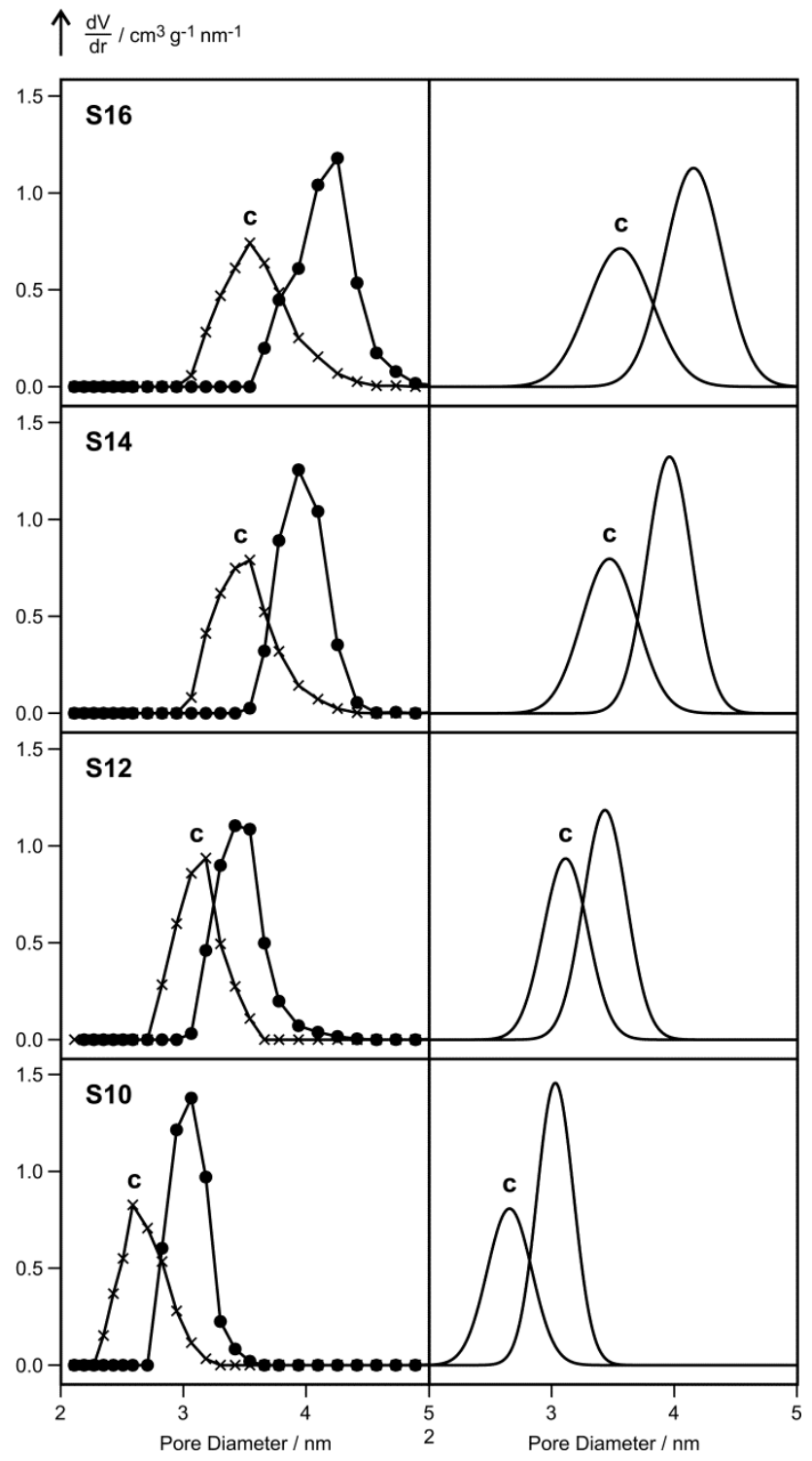

Figure 5. Left: PSDs of the materials obtained from Kromasil silica by pseudomorphic transformation with different alkyltrimethylammonium SDAs and subsequent removal by extraction (filled circles) or calcination (crosses). The right panels show the respective Gaussian fits which are used to estimate the FWHM.

\subsection{Incorporation of Amino Groups}

The pseudomorphic transformation as observed in the case of Kromasil silica entails the dissolution and reprecipitation of silica. Addition of APTES to the reaction mixture should therefore lead to the simultaneous inclusion of the aminopropyl moieties via hydrolysis of the alkoxysilyl groups, thereby 
producing a uniform distribution of amino groups over the entire surface area of the spherical particles. Our experiments reveal that this is indeed the case. Figure 6 shows as an example the PSDs of the pseudomorphically transformed samples S16A(0.10) and S16A(2.00). It should be mentioned that the NLDFT silica kernel used to calculate the PSDs is not strictly valid for the amino-functionalized materials. ${ }^{[33]}$ An analysis of the nitrogen adsorption isotherms by the BJH method, ${ }^{[34]}$ however, showed similar differences between the samples, although the BJH method is known to underestimate the pore diameter by up to $1 \mathrm{~nm} \cdot{ }^{[26,35-37]}$

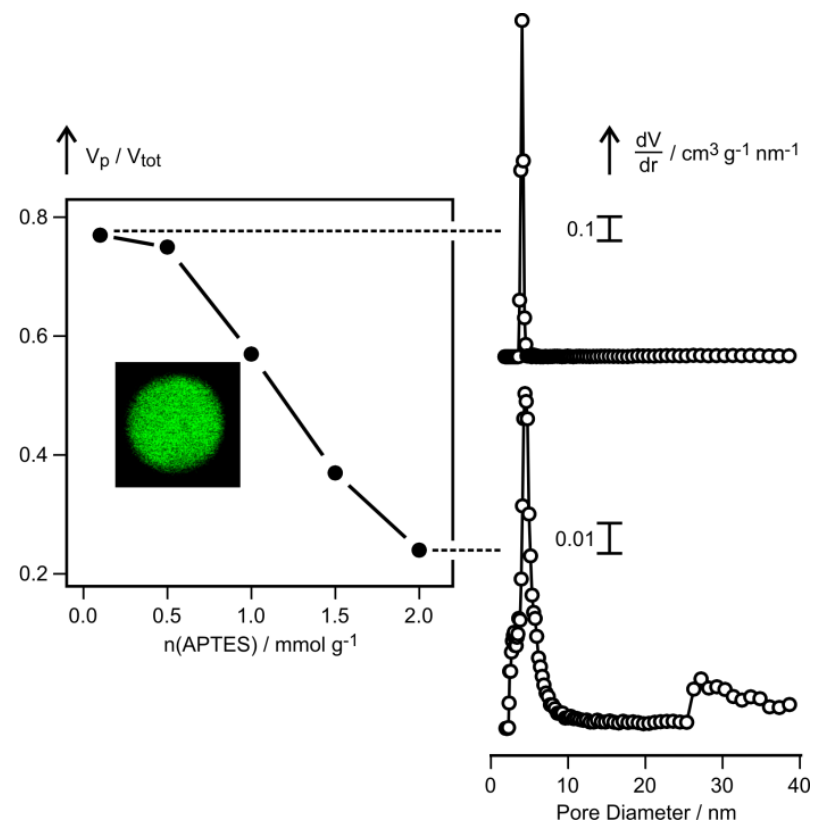

Figure 6. Ratio of the primary mesopore volume to the total pore volume as a function of the amount of APTES added to the pseudomorphic transformation with CTAB. The PSDs of S16A(0.10) and S16A(2.00) are given on the right. The inset shows a representative CLSM image of FITC-labeled S16A(1.00). An optical slice through the center of the microparticle was selected.

Narrow PSDs with predominant contributions from primary mesopores are obtained up to an added APTES amount of about $1 \mathrm{mmol}$ per gram of silica. Further increasing the APTES amount leads to a 
gradually decreasing fraction of the primary mesopore volume (Table 2, Figure 6) and a broadening of the PSD with the occurrence of various pore size regimes, indicating a decreasing degree of pseudomorphic transformation. From the results of the analysis of the incorporated amino groups, we can set the maximum functionalization degree to a value of approximately $1 \mathrm{mmol}$ of amino groups per gram of silica. The ratio of defined mesopores with pore sizes below $5 \mathrm{~nm}$ to larger mesopores and macropores can be controlled by the amount of APTES. This is of particular interest for applications in the fields of catalysis and chromatography, as the larger mesopores and macropores can provide access to the smaller mesopores, leading to favorable mass transfer kinetics. ${ }^{[23]}$

Table 2. Properties of the amino-functionalized materials prepared with CTAB.

\begin{tabular}{|c|c|c|c|c|c|c|c|c|}
\hline Sample & $\begin{array}{c}\mathrm{n}\left(\mathrm{NH}_{2}\right)^{\mathrm{a})} \\
{\left[\mathrm{mmol} \mathrm{g}^{-1}\right]}\end{array}$ & $\begin{array}{l}-\mathrm{NH}_{2}{ }^{\mathrm{a}}{ }^{2} \\
{\left[\mathrm{~nm}^{-2}\right]}\end{array}$ & $\begin{array}{l}\mathrm{d}_{\mathrm{DFT}}{ }^{\mathrm{b})} \\
{[\mathrm{nm}]}\end{array}$ & $\begin{array}{c}\left.\mathrm{FWHM}^{\mathrm{c}}\right) \\
{[\mathrm{nm}]}\end{array}$ & $\begin{array}{c}\mathrm{S}_{\mathrm{BET}} \\
{\left[\mathrm{m}^{2} \mathrm{~g}^{-1}\right]}\end{array}$ & $\begin{array}{c}V_{p} \\
{\left[\mathrm{~cm}^{3} \mathrm{~g}^{-1}\right]}\end{array}$ & $\begin{array}{c}V_{\text {tot }} \\
{\left[\mathrm{cm}^{3} \mathrm{~g}^{-1}\right]}\end{array}$ & $\mathrm{V}_{\mathrm{p}} / \mathrm{V}_{\text {tot }}$ \\
\hline $\mathrm{S} 16 \mathrm{~A}(0.10)$ & 0.03 & 0.03 & 4.25 & 0.37 & 647 & 0.53 & 0.69 & 0.77 \\
\hline $\mathrm{S} 16 \mathrm{~A}(0.50)$ & 0.33 & 0.31 & 4.20 & 0.41 & 639 & 0.52 & 0.69 & 0.75 \\
\hline S16A(1.00) & 0.57 & 0.84 & 4.31 & 0.72 & 411 & 0.32 & 0.56 & 0.57 \\
\hline $\mathrm{S} 16 \mathrm{~A}(1.50)$ & 0.96 & 1.57 & $4.61^{d)}$ & - & 368 & 0.22 & 0.60 & 0.37 \\
\hline $\mathrm{S} 16 \mathrm{~A}(2.00)$ & 0.99 & 1.91 & $4.67^{d)}$ & - & 312 & 0.13 & 0.55 & 0.24 \\
\hline
\end{tabular}

\footnotetext{
a) Amount of incorporated amino groups; ${ }^{\text {b) }}$ Average pore diameter of the primary mesopores determined by the NLDFT method; ${ }^{c)}$ Full width at half maximum of the primary mesopore size distribution; ${ }^{\text {d) }}$ Broad PSD.
}

CLSM has previously been used to assess the distribution of functional groups on mesoporous silica. ${ }^{[11,38-41]}$ CLSM images of the fluorescence-labeled amino-functionalized mesoporous silica particles feature a uniform distribution of labels throughout the particles, indicating a homogeneous distribution of accessible amino groups (Figure 6, inset). 
Amino-functionalized mesoporous silica spheres were also obtained with alkyltrimethylammonium SDAs other than CTAB. Pore size tuning was achieved to the same extent as in the purely siliceous materials (Table 3). Whereas the relative primary mesopore volume obtained by the pseudomorphic transformation is largely independent of the employed SDA in the case of the purely siliceous materials, the addition of APTES seems to affect the formation of the small mesopores. This is illustrated in Figure 7 by comparing samples obtained from the pseudomorphic transformation in the presence of $1 \mathrm{mmol}$ of APTES per gram of Kromasil silica. Despite the comparatively large amount of APTES, the use of CTAB yields a material with predominant primary mesopores and a narrow PSD around $4.3 \mathrm{~nm}$. However, with shorter SDAs, the pseudomorphic transformation becomes gradually less effective. This is in agreement with the observation of less ordered materials produced by shorter alkyltrimethylammonium SDAs due to the difficulty of self-organization. ${ }^{[42]}$ Analogous to the S16 samples shown in Figure 6, lowering of the APTES amount in the synthesis mixture increases the degree of pseudomorphic transformation for shorter SDAs. Similar to the pseudomorphic transformation without the addition of APTES, the spherical morphology and the particle size distribution are retained during the process, although a slight deterioration of the initially smooth external particle surface can be observed (Figure 7). 
Table 3. Properties of the amino-functionalized materials prepared with various SDAs.

\begin{tabular}{lcccccc}
\hline Sample & $\begin{array}{c}\mathrm{d}_{\mathrm{DFT}}{ }^{\mathrm{a}} \\
{[\mathrm{nm}]}\end{array}$ & $\begin{array}{c}\mathrm{FWHM}^{\mathrm{b})} \\
{[\mathrm{nm}]}\end{array}$ & $\begin{array}{c}\mathrm{S}_{\mathrm{BET}} \\
{\left[\mathrm{m}^{2} \mathrm{~g}^{-1}\right]}\end{array}$ & $\begin{array}{c}\mathrm{V}_{\mathrm{p}} \\
{\left[\mathrm{cm}^{3} \mathrm{~g}^{-1}\right]}\end{array}$ & $\begin{array}{c}\mathrm{V}_{\text {tot }} \\
{\left[\mathrm{cm}^{3} \mathrm{~g}^{-1}\right]}\end{array}$ & $\mathrm{V}_{\mathrm{p}} / \mathrm{V}_{\text {tot }}$ \\
\hline $\mathrm{S} 10 \mathrm{~A}(1.00)$ & $\left.-{ }^{\mathrm{c}}\right)$ & 2.24 & 147 & 0.02 & 0.71 & 0.03 \\
$\mathrm{~S} 12 \mathrm{~A}(1.00)$ & 3.53 & 1.58 & 223 & 0.08 & 0.66 & 0.12 \\
$\mathrm{~S} 14 \mathrm{~A}(1.00)$ & 3.85 & 0.63 & 334 & 0.21 & 0.62 & 0.34 \\
$\mathrm{~S} 16 \mathrm{~A}(1.00)$ & 4.31 & 0.72 & 411 & 0.32 & 0.56 & 0.57 \\
\hline
\end{tabular}

a) Average pore diameter of the primary mesopores determined by the NLDFT method; ${ }^{\text {b) }}$ Full width at half maximum of the primary mesopore size distribution; ${ }^{\mathrm{C})}$ Broad PSD with dominant contribution from parental pores.
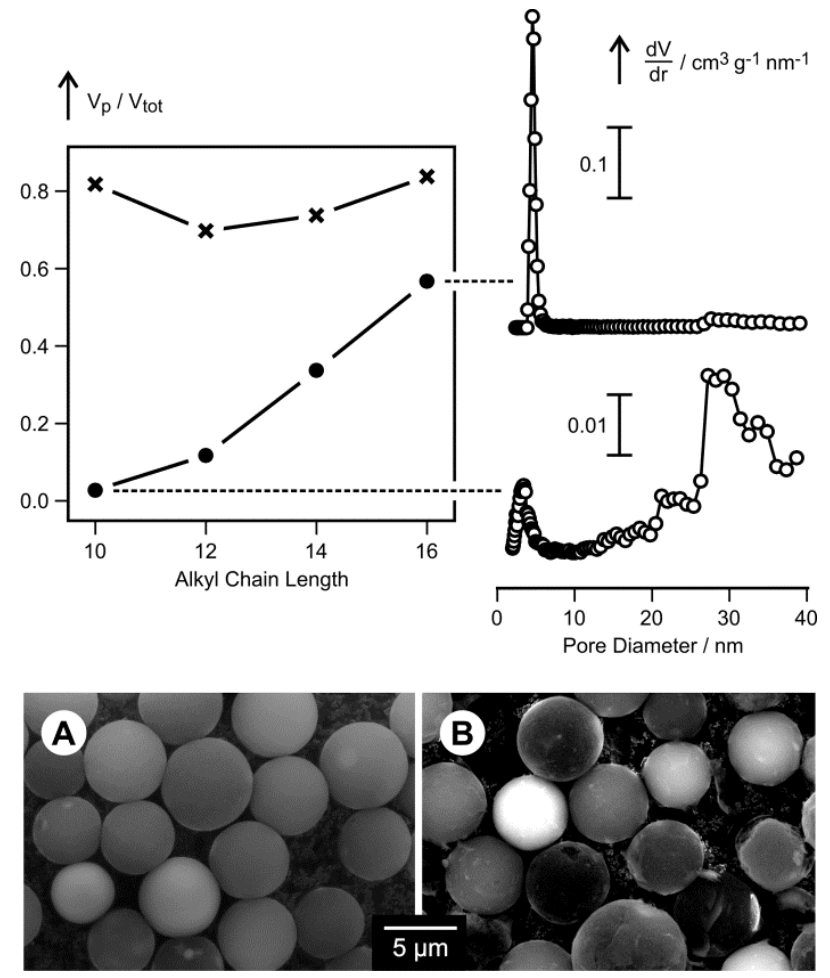

Figure 7. Ratio of the primary mesopore volume to the total pore volume as a function of the alkyl chain length of the SDA for purely siliceous samples (crosses) and samples synthesized with addition of $1 \mathrm{mmol}$ of APTES per gram of Kromasil silica (filled circles). The PSDs of S16A(1.00) and S10A(1.00) are given on the right. The SEM images show the spherical particles of the parent Kromasil sample (image A) and of S16A(1.00) (image B). 


\section{Conclusion}

The synthesis of well-defined non-agglomerated mesoporous silica microspheres by pseudomorphic transformation can be combined with pore size control and co-condensation approaches. The addition of APTES to the mixture of parent silica, SDA, and aqueous alkaline solution yields a uniformly aminofunctionalized mesoporous material with a narrow pore size distribution and fully accessible pores. The particle size and morphology of the starting material are conserved during the transformation. Apart from acting as the functionalization agent, APTES can be used to control the porosity of the products over a wide range. In particular, this concerns the ratio of small uniform mesopores to larger mesopores and macropores. The combination of small mesopores (providing a large surface area) with macropores (providing access to the mesopores) is advantageous, especially in micrometer-sized particles, where an efficient mass transfer is considered an important prerequisite for the application of these materials in separation processes and catalysis.

\section{Experimental Section}

Pseudomorphic Transformation: Kromasil silica (300 $\AA, 5 \mu \mathrm{m}$, AkzoNobel) was used as the starting material for the pseudomorphic transformation, following a previously reported procedure based on hexadecyltrimethylammonium bromide $(\mathrm{CTAB})$ as $\mathrm{SDA} .{ }^{[43]}$ The reactants $\mathrm{SiO}_{2}: \mathrm{NaOH}: \mathrm{SDA}: \mathrm{H}_{2} \mathrm{O}$ with a molar composition of 1:0.25:0.1:20 were stirred for $30 \mathrm{~min}$ at room temperature. The suspension was then transferred to a Teflon lined autoclave, where it was kept at $100{ }^{\circ} \mathrm{C}$ for $24 \mathrm{~h}$. The product was filtered and washed with $250 \mathrm{~mL}$ of $\mathrm{H}_{2} \mathrm{O}$. The SDA was removed by calcination $\left(550{ }^{\circ} \mathrm{C}\right.$ for $5 \mathrm{~h}$ after $12 \mathrm{~h}$ of drying at $80^{\circ} \mathrm{C}$ and a heating rate of $1{ }^{\circ} \mathrm{C} \mathrm{min}$ ) or extraction with an ethanolic solution of ammonium nitrate according to reference 44 (three times with $50 \mathrm{~mL}$ ). The product was filtered and washed with $250 \mathrm{~mL}$ of $\mathrm{H}_{2} \mathrm{O}$. A typical batch consisted of $300 \mathrm{mg}$ of Kromasil silica. 
The following SDAs were used: Decyltrimethylammonium bromide (Aldrich, $\geq 98 \%$ ), dodecyltrimethylammonium bromide (Fluka, $\geq 98 \%$ ), tetradecyltrimethylammonium bromide (Sigma, $\geq 99 \%$ ), and hexadecyltrimethylammonium bromide (CTAB, Fluka, $\geq 96 \%)$. The synthesis of the amino-functionalized materials followed the above procedure for the pseudomorphic transformation of Kromasil silica. Varying amounts of 3-aminopropyltriethoxysilane (APTES, Fluka, $\geq 98 \%, 0.1$ to $2.0 \mathrm{mmol}$ per gram of Kromasil silica) were added to the SDA/Kromasil suspension after dissolution of the respective SDA.

Functionalization and Fluorescent Labeling: Functionalization of Kromasil silica or pseudomorphically transformed samples was performed according to reference 11. Typically, an amount of 20-200 mg of silica was dispersed in dry toluene $(5-10 \mathrm{~mL}$ ) and $10 \mu \mathrm{mol}$ of APTES (Fluka, $\geq 98 \%$ ) or 3-aminopropyltris(methoxyethoxyethoxy)silane (APTMEES, ABCR Karlsruhe, $95 \%$ ) per $100 \mathrm{mg}$ of silica was added. After stirring for $10 \mathrm{~min}$, the silica was recovered by filtration and cured in an oven at $80{ }^{\circ} \mathrm{C}$ for $16 \mathrm{~h}$. Samples that were amino-functionalized, either postsynthetically or by co-condensation, were fluorescence labeled by stirring in ethanol containing 1.5 equivalents (relative to the amount of the maximum theoretical amino group content) of fluorescein 5-isothiocyanate (FITC, Sigma, $\geq 97.5 \%$ ) or rhodamine B isothiocyanate (RBITC, Aldrich, mixed isomers) for $16 \mathrm{~h}$ at room temperature. The labeled samples were repeatedly washed with ethanol until the washing solution became colorless.

Amino Group Analysis: The amount of amino groups introduced into the mesoporous silica framework was analyzed according to a previously published method based on the fluorometric quantitation of primary amino groups with fluorescamine. ${ }^{[45-47]}$ An amount of $15 \mathrm{mg}$ of amino-functionalized mesoporous silica was stirred in $30 \mathrm{~mL}$ of $0.02 \mathrm{M}$ aqueous $\mathrm{NaOH}$ until dissolved. A $100 \mu \mathrm{L}$ aliquot of this solution was transferred into a cuvette $(\mathrm{d}=1 \mathrm{~cm})$ and $2 \mathrm{~mL}$ of phosphate buffer $(0.2 \mathrm{M}, \mathrm{pH} 8.0)$ was added. After the addition of $1 \mathrm{~mL}$ of fluorescamine (Sigma, $\geq 98 \%$ ) solution ( $1 \mathrm{mM}$ in acetone), the fluorescence spectrum was measured by excitation at $366 \mathrm{~nm}$. The emission intensity at $480 \mathrm{~nm}$ was taken as the data point. A calibration line was prepared accordingly by using $100 \mu \mathrm{L}$ aliquots of 
differently concentrated solutions of APTES in $30 \mathrm{~mL}$ of $0.02 \mathrm{M}$ aqueous $\mathrm{NaOH}$ (containing $15 \mathrm{mg}$ of S16). Repeated analysis of the same sample gave an average relative error of $5 \%$ (for amino contents in the range of 0.03-0.99 $\mathrm{mmol} \mathrm{g}^{-1}$ ).

Physical Measurements: Nitrogen sorption isotherms were measured at $77 \mathrm{~K}$ with a Quantachrome NOVA 3000. Samples were vacuum-degassed at $80{ }^{\circ} \mathrm{C}$ for $3 \mathrm{~h}$ prior to the measurement. Pore size distributions and total surface areas $\left(\mathrm{S}_{\mathrm{BET}}\right)$ were determined by $\mathrm{NLDFT}^{[33]}$ and $\mathrm{BET}^{[48]}$ methods, respectively. Primary mesopore volumes $\left(V_{p}\right)$ were determined from the $\alpha_{s}$-plots. ${ }^{[49,50]}$ Total pore volumes $\left(V_{\text {tot }}\right)$, including all pores with diameters below $80 \mathrm{~nm}$, were calculated from the NLDFT analysis. In agreement with reference 35, adsorption isotherms were used for all calculations. A Perkin-Elmer LS55 spectrofluorometer was used for the fluorescamine assays. Scanning electron microscopy (SEM) was performed with a FEI Quanta FEG 250. Particle size distributions were determined with ImageJ (Version 1.48s, National Institutes of Health, USA) by counting at least 400 particles in random fields of view of at least four SEM images. The fluorescence and CLSM setup consisted of an Olympus BX60 microscope equipped with a FluoView FV300 confocal unit and lasers operating at $488 \mathrm{~nm}$ and $543.5 \mathrm{~nm}$.

Acknowledgements. Financial support by the Swiss National Science Foundation (Project 140303) is acknowledged. We thank Dr. Andrei Honciuc and Dr. Emmanouil Tzirakis for SEM imaging assistance. 


\section{References}

[1] P. Yang, S. Gai, J. Lin, Chem. Soc. Rev. 2012, 41, 3679.

[2] C. Coll, A. Bernardos, R. Martinez-Mañez, F. Sancenón, Acc. Chem. Res. 2013, 46, 339.

[3] J. H. Clark, D. J. Macquarrie, S. J. Tavener, Dalton Trans. 2006, 4297.

[4] H. Yoshitake, J. Mater. Chem. 2010, 20, 4537.

[5] S. Li, X. Jiao, H. Yang, Langmuir 2013, 29, 1228.

[6] C. Argyo, V. Weiss, C. Bräuchle, T. Bein, Chem. Mater. 2014, 26, 435.

[7] D. Tarn, C. E. Ashley, M. Xue, E. C. Carnes, J. I. Zink, C. J. Brinker, Acc. Chem. Res. 2013, 46, 792.

[8] H. Meng, M. Xue, T. Xia, Z. Ji, D. Y. Tarn, J. I. Zink, A. E. Nel, ACS Nano 2011, 5, 4131.

[9] T. Yu, D. Hubbard, A. Ray, H. Ghandehari, J. Control. Release 2012, 163, 46.

[10] N. Linares, E. Serrano, M. Rico, A. M. Balu, E. Losada, R. Luque, J. Garcia-Martinez, Chem. Commun. 2011, $47,9024$.

[11] N. Gartmann, D. Brühwiler, Angew. Chem. Int. Ed. 2009, 48, 6354.

[12] D. Brühwiler, Nanoscale 2010, 2, 887.

[13] H. Ritter, D. Brühwiler, J. Phys. Chem. C 2009, 113, 10667.

[14] S. Huh, J. W. Wiench, B. G. Trewyn, S. Song, M. Pruski, V. S.-Y. Lin, Chem. Commun. 2003, 2364.

[15] K. Möller, J. Kobler, T. Bein, J. Mater. Chem. 2007, 17, 624.

[16] F. O. M. Gaslain, C. Delacôte, A. Walcarius, B. Lebeau, J. Sol-Gel Sci. Technol. 2009, 49, 112.

[17] T. Martin, A. Galarneau, F. Di Renzo, F. Fajula, D. Plee, Angew. Chem. Int. Ed. 2002, 41, 2590.

[18] A. Galarneau, J. Iapichella, K. Bonhomme, F. Di Renzo, P. Kooyman, O. Terasaki, F. Fajula, Adv. Funct. Mater. 2006, 16, 1657.

[19] J. Patzsch, J. J. Schneider, Dalton Trans. 2013, 42, 1451.

[20] T. Yasmin, K. Müller, J. Chromatogr. A 2010, 1217, 3362.

[21] Y. Wan, D. Zhao, Chem. Rev. 2007, 107, 2821.

[22] B. Lefèvre, A. Galarneau, J. lapichella, C. Petitto, F. Di Renzo, F. Fajula, Z. Bayram-Hahn, R. Skudas, K. Unger, Chem. Mater. 2005, 17, 601.

[23] M. Grün, A. A. Kurganov, S. Schacht, F. Schüth, K. K. Unger, J. Chromatogr. A 1996, 740, 1.

[24] M. Grün, I. Lauer, K. K. Unger, Adv. Mater. 1997, 9, 254.

[25] A. Kierys, W. Buda, J. Goworek, J. Porous Mater. 2010, 17, 669.

[26] M. Kruk, M. Jaroniec, A. Sayari, J. Phys. Chem. B 1997, 101, 583.

[27] H.-P. Lin, S. Cheng, C.-Y. Mou, Microporous Mater. 1997, 10, 111.

[28] L. Martins, D. Cardoso, Microporous Mesoporous Mater. 2007, 106, 8.

[29] R. Ji, K. Yu, L.-L. Lou, Z. Gu, S. Liu, J. Inorg. Organomet. Polym. 2010, 20, 675.

[30] M. Fujiwara, F. Yamamoto, K. Okamoto, K. Shiokawa, R. Nomura, Anal. Chem. 2005, 77, 8138.

[31] X. Hu, M. L. Foo, G. K. Chuah, S. Jaenicke, J. Catal. 2000, 195, 412.

[32] D. Brühwiler, H. Ritter, J. H. Ramm, L.-Q. Dieu, C. Bauer, I. Dolamic, N. Gartmann, Chimia 2009, 63, 8.

[33] J. Landers, G. Y. Gor, A. V. Neimark, Colloids Surf. A: Physicochem. Eng. Aspects 2013, 437, 3.

[34] E. P. Barrett, L. G. Joyner, P. P. Halenda, J. Am. Chem. Soc. 1951, 73, 373.

[35] P. I. Ravikovitch, A. V. Neimark, Colloids Surf. A: Physicochem. Eng. Aspects 2001, 187-188, 11. 
[36] P. I. Ravikovitch, D. Wei, W. T. Chueh, G. L. Haller, A. V. Neimark, J. Phys. Chem. B 1997, 101, 3671.

[37] M. A. Smith, M. G. Ilasi, A. Zoelle, J. Phys. Chem. C 2013, 117, 17493.

[38] N. Gartmann, C. Schütze, H. Ritter, D. Brühwiler, J. Phys. Chem. Lett. 2010, 1, 379.

[39] J. H. Ramm, N. Gartmann, D. Brühwiler, J. Colloid Interf. Sci. 2010, 345, 200.

[40] N. Gartmann, D. Brühwiler, Materials 2011, 4, 1096.

[41] K. Cheng, C. C. Landry, J. Am. Chem. Soc. 2007, 129, 9674.

[42] A. Sayari, Y. Yang, J. Phys. Chem. B 2000, 104, 4835.

[43] K. Kailasam, A. Fels, K. Müller, Microporous Mesoporous Mater. 2009, 117, 136.

[44] N. Lang, A. Tuel, Chem. Mater. 2004, 16, 1961.

[45] H. Ritter, M. Nieminen, M. Karppinen, D. Brühwiler, Microporous Mesoporous Mater. 2009, 121, 79.

[46] M. Weigele, S. L. De Bernardo, J. P. Tengi, W. Leimgruber, J. Am. Chem. Soc. 1972, 94, 5927.

[47] S. De Bernardo, M. Weigele, W. Toome, K. Manhart, W. Leimgruber, P. Böhlen, S. Stein, S. Udenfriend, Arch. Biochem. Biophys. 1974, 163, 390.

[48] S. Brunauer, P. H. Emmett, E. Teller, J. Am. Chem. Soc. 1938, 60, 309.

[49] M. Kruk, M. Jaroniec, R. Ryoo, J. M. Kim, Microporous Mater. 1997, $12,93$.

[50] A. Sayari, P. Liu, M. Kruk, M. Jaroniec, Chem. Mater. 1997, 9, 2499. 